\title{
Dexmedetomidine for sedation of patients undergoing elective surgery under regional anesthesia
}

\author{
Jia Song, Woong-Mo Kim, Seong-Heon Lee, and Myung Ha Yoon \\ Department of Anesthesiology and Pain Medicine, Chonnam National University Hospital, Gwangju, Korea
}

Background: Dexmedetomidine may be useful as a sedative agent. However, it has been reported that dexmedetomidine decreases systemic blood pressure, heart rate, and cardiac output in a dose-dependent manner. The purpose of this study was to determine the appropriate dose of intravenously administered dexmedetomidine for sedation.

Methods: Forty-five American Society of Anesthesiologists physical status I-II patients under spinal anesthesia received dexmedetomidine $1 \mu \mathrm{g} / \mathrm{kg}$ intravenously as a loading dose. The patients were randomly allocated to one of three groups for maintenance dose: Group A $(0.25 \mu \mathrm{g} / \mathrm{kg} / \mathrm{hr})$, Group B $(0.50 \mu \mathrm{g} / \mathrm{kg} / \mathrm{hr})$, and Group C $(0.75 \mu \mathrm{g} / \mathrm{kg} / \mathrm{hr})$. The hemodynamic variables and the Ramsay Sedation Scale (RSS) score were recorded for all patients. The numbers of patients who developed hypotension, bradycardia, or inadequate sedation necessitating further drug treatment were also recorded.

Results: Systolic blood pressure, heart rate, and $\mathrm{SpO}_{2}$ were decreased, and RSS score was increased significantly at both $20 \mathrm{~min}$ and $40 \mathrm{~min}$ after injection of dexmedetomidine in the three study groups compared to baseline, without significant differences between the groups. The prevalence of hypotension, but not that of bradycardia or adjunctive midazolam administration, exhibited a positive correlation with the dose of dexmedetomidine.

Conclusions: Intravenous injection of dexmedetomidine $1 \mu \mathrm{g} / \mathrm{kg}$ followed by continuous administration at infusion rates of $0.25,0.50$, or $0.75 \mu \mathrm{g} / \mathrm{kg} / \mathrm{hr}$ produced adequate levels of sedation. However, there was a tendency for the incidence of hypotension to increase as the dose increased. To minimize the risk of hemodynamic instability, a dose of $0.25 \mu \mathrm{g} / \mathrm{kg} / \mathrm{hr}$ may be the most appropriate for continuous administration of dexmedetomidine. (Korean J Anesthesiol 2013; 65: 203-208)

Key Words: Continuous dose, Dexmedetomidine, Regional anesthesia, Sedation.

\footnotetext{
Received: July 4, 2012. Revised: 1st, October 22, 2012; 2nd, November 29, 2012; 3rd, January 12, 2013; 4th, January 27, 2013. Accepted: February 8, 2013. Corresponding author: Myung Ha Yoon, M.D., Department of Anesthesiology and Pain Medicine, Chonnam National University Medical School, 671, Jebong-ro, Dong-gu, Gwangju 501-757, Korea. Tel: 82-62-220-6893, Fax: 82-62-232-6294, E-mail: mhyoon@chonnam.ac.kr (c) This is an open-access article distributed under the terms of the Creative Commons Attribution Non-Commercial License (http:// creativecommons.org/licenses/by-nc/3.0/), which permits unrestricted non-commercial use, distribution, and reproduction in any medium, provided the original work is properly cited.
} 


\section{Introduction}

Spinal anesthesia offers a number of advantages to both the patient and the physician. Among these advantages are spared spontaneous respiration, reduced risk of pulmonary aspiration secondary to vomiting in patients with a full stomach, facilitated surgery via provision of relaxation in the intestines and abdominal wall, elimination of the need for intubation, minimal disruption of blood chemistry, reduced surgical hemorrhage, and earlier return of intestinal motility [1]. However, patients are often reluctant to remain awake during a procedure and the requirement to maintain uncomfortable positioning throughout a surgery of long duration can cause spontaneous movements which may interfere with the surgical procedure [2]. Therefore, provision of adequate sedation is important if the advantages of spinal anesthesia are to be fully appreciated. Several agents including midazolam, ketamine, remifentanil, propofol, and dexmedetomidine have been used for this purpose.

Among these agents, dexmedetomidine is a centrally acting $\alpha-2$ adrenoceptor agonist that can be titrated to the desired level of sedation without causing significant respiratory depression [3-5]. Dexmedetomidine has an analgesic-sparing effect, significantly reducing opioid requirements both during and after surgery [6-8]. Because of its analgesic properties and lack of respiratory depression as described above, dexmedetomidine is becoming increasingly used as a sedative agent [3]. On the other hand, dexmedetomidine has been seen to exhibit a sympatholytic effect [7] and has also been reported to decrease systemic blood pressure, heart rate, and cardiac output in a dose-dependent manner [9]. Therefore, the dose of dexmedetomidine which will not cause hemodynamic instability, while at the same time producing a sufficient level of sedation, should be selected. However, to the author's knowledge, the adequate dose of dexmedetomidine for sedation during spinal anesthesia has not been previously investigated.

In this study, the primary goal was to determine the appropriate dose of intravenously administered dexmedetomidine for sedation in spinal anesthesia, minimizing the possibility of hemodynamic instability.

\section{Materials and Methods}

After approval was received from the Institutional Review Board, informed consent was obtained from each patient. Forty-five patients who were classified as American Society of Anesthesiologists physical status I-II, scheduled for lower extremity or urogenital surgery, were enrolled in this study. Exclusion criteria included patients with hypovolemia, coagulation disorders, local infection at the site of operation, history of headache, pregnancy, heart diseases, and history of allergy, chronic alcohol use or abuse, anemia, and patients who had recently received sedative drugs or were under antidepressant treatment. All patients arrived in the operating room without premedication and were monitored with noninvasive arterial blood pressure, electrocardiogram, heart rate, and pulse oximetry. After intravenous hydration with $300 \mathrm{ml}$ of Ringer's lactate solution via an 18-gauge cannula in the dorsum of the hand, spinal anesthesia was performed. With the patient in the lateral decubitus position, the subarachnoid space was punctured at the L4-L5 interlaminar space by a midline approach using a 25-gauge Quincke spinal needle (B. Braun Medical, Melsungen, Germany) with the hole pointing upwards. If the spinal block failed at the L4-L5 level, another puncture was attempted at L3-L4. In case of failure at both levels, the surgery proceeded under general anesthesia and the patient was excluded from the study protocol. In the other cases, after the visualization of clear flow of cerebrospinal fluid, $2 \mathrm{ml}$ of $0.5 \%$ hyperbaric bupivacaine was injected at a rate of $1 \mathrm{ml}$ per 3 seconds, the patients were brought to the supine position, and oxygen $5 \mathrm{~L} / \mathrm{min}$ was administered by simple face mask.

Thereafter, intravenous administration of dexmedetomidine (Precedex ${ }^{\circledR}$, Hospira Inc., Lake Forest, USA) diluted with normal saline to a concentration of $4 \mu \mathrm{g} / \mathrm{ml}$ was started. After injection of $1 \mu \mathrm{g} / \mathrm{kg}$ dexmedetomidine over 10 minutes as a loading dose, patients were allocated to one of three groups of maintenance dose of intravenous dexmedetomidine: Group A received $0.25 \mu \mathrm{g} / \mathrm{kg} / \mathrm{hr}$, Group B received $0.50 \mu \mathrm{g} / \mathrm{kg} / \mathrm{hr}$, and Group C received $0.75 \mu \mathrm{g} / \mathrm{kg} / \mathrm{hr}$. The dose of continuous intravenous dexmedetomidine in each group was based on a previous study in which the intended level of sedation was achieved at a dose of $0.2-0.7 \mu \mathrm{g} / \mathrm{kg} / \mathrm{hr}$ [10], and equal spacing was determined upon to facilitate statistical comparison. The anesthesiologist was blinded to patient group allocation. Surgical procedures were initiated in the study patients when sensory blockade was achieved up to the T10 dermatome bilaterally.

The systolic blood pressure, heart rate, peripheral oxygen saturation, and level of sedation were recorded before spinal anesthesia: immediately before, 20, 40, and $60 \mathrm{~min}$ after the administration of dexmedetomidine: and in the post-anesthesia care unit (PACU). The level of sedation was evaluated using the Ramsay Sedation Scale (RSS) [11]: 1, Patient anxious, agitated, or restless; 2, Patient cooperative, oriented, and tranquil alert; 3 , Patient responds to commands; 4, Asleep, but with brisk response to light glabellar tap or loud auditory stimulus; 5, Asleep, sluggish response to light glabellar tap or loud auditory stimulus; 6 , Asleep, no response. Before skin incision, about twenty minutes after dexmedetomidine infusion, any patient having a RSS score $<3$ received IV midazolam in $0.5 \mathrm{mg}$ doses, repeated until the patient exhibited a RSS score $\geq 3$. Hypotension was defined as systolic blood pressure of less than $90 \mathrm{mmHg}$ or decrease of 
$30 \%$ from baseline and was treated with a bolus administration of $300 \mathrm{ml}$ of Ringer's lactate solution over $10 \mathrm{~min}$ and $6 \mathrm{mg}$ of intravenous ephedrine. Bradycardia was defined as heart rate $<50$ beats/min and treated with $0.5 \mathrm{mg}$ of intravenous atropine. The incidence of administration of midazolam, ephedrine, or atropine, and the time from arrival to discharge from PACU were also recorded.

Sample size calculation for this study was based on a pilot study in which the incidence of hypotension or bradycardia after dexmedetomidine administration was 0,10 , and $40 \%$ in groups A, B, and C, respectively. A sample size of 15 patients in each group was obtained to achieve a power above $80 \%$ to detect a linear trend at a two-sided significance level of 0.05 , using sample size software (PASS 11; NCCS, Kaysville, UT). Data are expressed as mean \pm standard deviation. The demographic data of patients with parametric data were analyzed using one-way analysis of variance (ANOVA) and RSS scores were compared with the Kruskal-Wallis test. Repeated measures ANOVA was utilized to compare data over time. Incidence variables were compared using the Chi-square test, and their relationship with

Table 1. Demographic Characteristics of Patients

\begin{tabular}{lccc}
\hline & $\begin{array}{c}\text { Group A } \\
(\mathrm{n}=15)\end{array}$ & $\begin{array}{c}\text { Group B } \\
(\mathrm{n}=15)\end{array}$ & $\begin{array}{c}\text { Group C } \\
(\mathrm{n}=15)\end{array}$ \\
\hline Gender $(\mathrm{M} / \mathrm{F})$ & $12 / 3$ & $13 / 2$ & $10 / 5$ \\
Age $(\mathrm{yr})$ & $55.86 \pm 24.30$ & $50.27 \pm 19.72$ & $52.53 \pm 14.91$ \\
Weight $(\mathrm{kg})$ & $68.50 \pm 12.08$ & $66.40 \pm 12.15$ & $63.33 \pm 11.19$ \\
Height $(\mathrm{cm})$ & $165.64 \pm 8.37$ & $166.27 \pm 9.43$ & $165.07 \pm 11.81$ \\
\hline
\end{tabular}

Values are mean \pm SD or number of patients. Groups $A, B$, and $C$ received dexmedetomidine $0.25,0.50$, and $0.75 \mu \mathrm{g} / \mathrm{kg} / \mathrm{hr}$, respectively, after bolus administration of $1 \mu \mathrm{g} / \mathrm{kg}$.

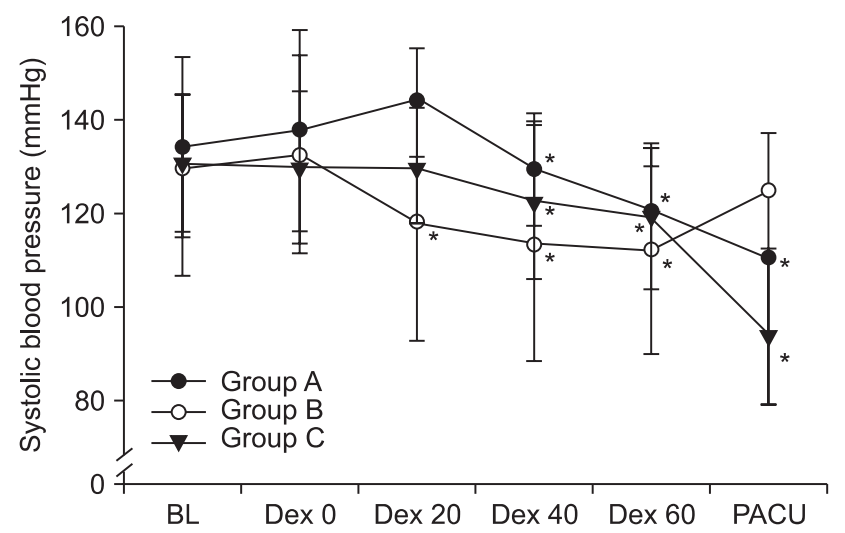

Fig. 1. Systolic blood pressure in the study groups. Systolic blood pressure is significantly decreased from $20 \mathrm{~min}$ or $40 \mathrm{~min}$ after injection of dexmedetomidine in the three study groups compared to baseline without significant difference between groups. BL: baseline value before spinal anesthesia, Dex 0, 20, 40, and 60: immediately before, 20, 40, and 60 min after the administration of dexmedetomidine, PACU: postanesthesia care unit. $* \mathrm{P}<0.05$ compared to baseline. the dose of dexmedetomidine was measured with Spearman's rank correlation to test a linear trend. Statistical analysis was performed using SPSS software (version 17.0; SPSS Inc., Chicago, IL, USA). P values of $<0.05$ were considered significant.

\section{Results}

All forty-five patients who were enrolled in the study completed the study protocol and are included in the data analysis. No spinal anesthesia failure occurred. Thus each group consisted of 15 patients. Demographic data did not differ among the three study groups (Table 1). No significant hemodynamic changes

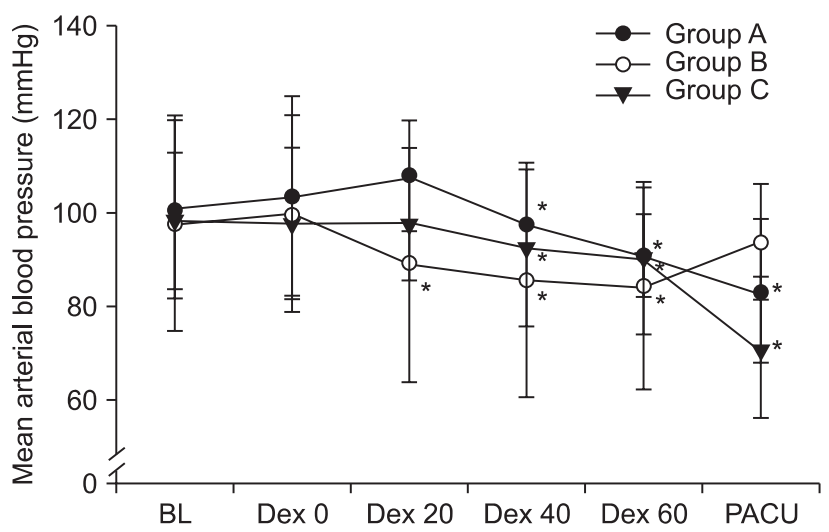

Fig. 2. Mean arterial blood pressure in the study groups. Mean arterial blood pressure is significantly decreased from $20 \mathrm{~min}$ or $40 \mathrm{~min}$ after injection of dexmedetomidine in the three study groups compared to baseline without significant difference between groups. BL: baseline value before spinal anesthesia, Dex $0,20,40$, and 60: immediately before, 20,40 , and $60 \mathrm{~min}$ after the administration of dexmedetomidine, PACU: post-anesthesia care unit. ${ }^{*} \mathrm{P}<0.05$ compared to baseline.

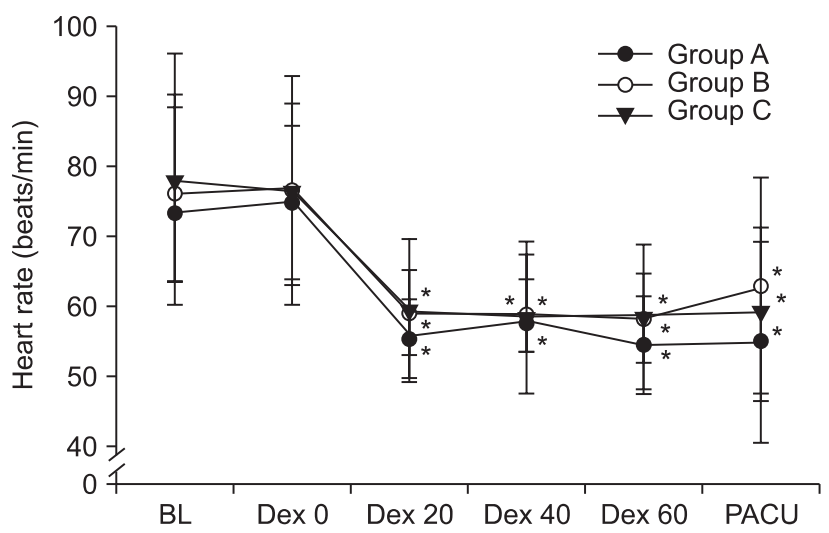

Fig. 3. Heart rate values in the study groups. Heart rate is significantly decreased from $20 \mathrm{~min}$ after injection of dexmedetomidine in the three study groups compared to baseline without significant difference between groups. BL: baseline value before spinal anesthesia, Dex 0, 20, 40, and 60: immediately before, 20, 40, and $60 \mathrm{~min}$ after the administration of dexmedetomidine, PACU: post-anesthesia care unit. $* \mathrm{P}<0.05$ compared to baseline. 


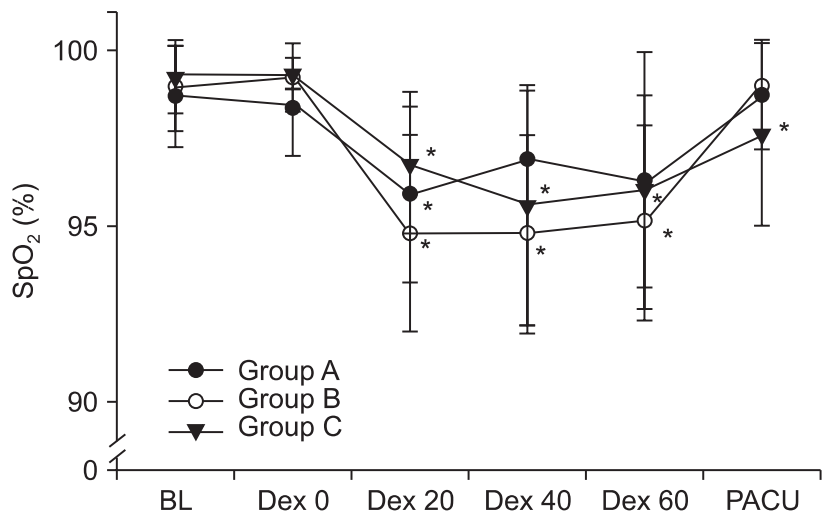

Fig. 4. $\mathrm{SpO}_{2}$ values in the study groups. $\mathrm{SpO}_{2}$ is significantly decreased from $20 \mathrm{~min}$ after injection of dexmedetomidine in the three study groups compared to baseline without significant difference between groups. BL: baseline value before spinal anesthesia, Dex 0, 20, 40, and 60: immediately before, 20,40 , and 60 min after the administration of dexmedetomidine, PACU: post-anesthesia care unit. ${ }^{*} \mathrm{P}<0.05$ compared to baseline.

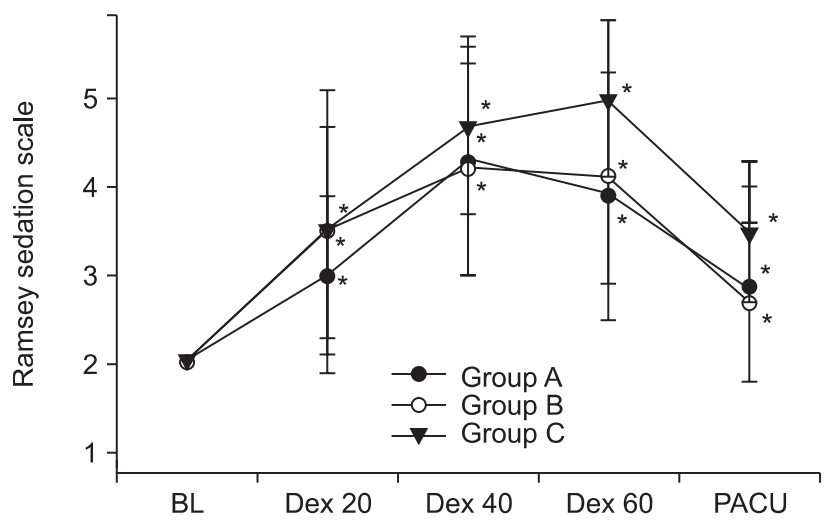

Fig. 5. RSS scores in the study groups. RSS score is significantly increased from $20 \mathrm{~min}$ after injection of dexmedetomidine in the three study groups compared to baseline without significant difference between groups. RSS: Ramsay Sedation Scale, BL: baseline value before spinal anesthesia, Dex 0, 20, 40, and 60: immediately before, 20, 40, and $60 \mathrm{~min}$ after the administration of dexmedetomidine, PACU: postanesthesia care unit. $* \mathrm{P}<0.05$ compared to baseline.

were observed between baseline and immediately before the administration of dexmedetomidine in the three groups. Compared to baseline, systolic blood pressure, heart rate, and $\mathrm{SpO}_{2}$ were decreased, and RSS score was increased significantly from 20 min after injection of dexmedetomidine in the three study groups. However, the change in those variables over time showed no statistically significant differences between the groups (Fig. 1-5). In group A, no patient developed clinically significant hypotension necessitating drug treatment. Three patients (20\%) in group B and 5 patients (33.3\%) in group C developed hypotension after dexmedetomidine administration, but intravenous ephedrine injection restored the blood pressure to the normal range. The prevalence of hypotension exhibited
Table 2. Distribution of Side Effects

\begin{tabular}{llccc}
\hline & $\begin{array}{c}\text { Group A } \\
\mathrm{n}(\%)\end{array}$ & $\begin{array}{c}\text { Group B } \\
\mathrm{n}(\%)\end{array}$ & $\begin{array}{c}\text { Group C } \\
\mathrm{n}(\%)\end{array}$ & $\begin{array}{c}\text { Spearman's } \\
\text { correlation/P* }\end{array}$ \\
\hline Bradycardia & $5(33.3)$ & $2(13.3)$ & $6(40.0)$ & $0.046 / 0.77$ \\
Hypotension & $0(0)$ & $3(20.0)$ & $5(33.3)$ & $0.356 / 0.02$ \\
Need to midazolam & $4(26.7)$ & $4(26.7)$ & $3(20.0)$ & $-0.080 / 0.60$ \\
\hline
\end{tabular}

Groups A, B, and C received dexmedetomidine $0.25,0.5$, and $0.75 \mu \mathrm{g} / \mathrm{kg} /$ $\mathrm{hr}$, respectively, after bolus administration of $1 \mu \mathrm{g} / \mathrm{kg}$. The prevalence of hypotension exhibited a positive correlation with the dose of dexmedetomidine. ${ }^{*} \mathrm{P}<0.05$ compared to baseline.

a positive correlation with the dose of dexmedetomidine in groups $\mathrm{A}, \mathrm{B}$, and $\mathrm{C}$ (Spearman's rank correlation coefficient $=$ $0.36, \mathrm{P}=0.02$, Table 2 ). The incidence of bradycardia requiring atropine administration and numbers of patients requiring midazolam in each group were comparable between the three groups and showed no statistically significant correlation with the dose of dexmedetomidine (Table 2).

Although a statistically significant change in $\mathrm{SpO}_{2}$ was apparent after administration of dexmedetomidine, clinically significant desaturation below $90 \%$ was not observed in patients in any of the three study groups.

\section{Discussion}

In this study, intravenous injection of dexmedetomidine $1 \mu \mathrm{g} / \mathrm{kg}$ followed by continuous administration at an infusion rate of $0.25,0.50$, or $0.75 \mu \mathrm{g} / \mathrm{kg} / \mathrm{hr}$ produced an adequate level of sedation without any significant differences among the groups. However, there was a tendency for the incidence of hypotension, but not of bradycardia or desaturation, to increase with increasing dose of dexmedetomidine. These findings suggest that continuous administration of dexmedetomidine $0.25 \mu \mathrm{g} / \mathrm{kg} / \mathrm{hr}$ after bolus injection of $1 \mu \mathrm{g} / \mathrm{kg}$ may be an appropriate dose for providing an adequate level of sedation while minimizing the risk of hemodynamic instability in patients undergoing elective surgery of lower extremitie or urogenital organs under spinal anesthesia.

Dexmedetomidine is an $\alpha-2$ adrenoceptor agonist. It produces sedation and anxiolysis by binding to $\alpha-2$ adrenoceptors in the locus ceruleus, which diminishes the release of norepinephrine and inhibits sympathetic activity, thus decreasing heart rate and blood pressure [12]. Consequently, it has been reported that the most common adverse effects after dexmedetomidine administration are hypotension and bradycardia, although treatment with a vasopressor drug restored both parameters to the normal range $[9,13]$. In the current study, the prevalence of hypotension requiring drug treatment exhibited a positive correlation with the dose of dexmedetomidine in all three groups, and this finding is in line with a previous report using bolus administration of dexmedetomidine $0.25,0.5,1$, or 2 
$\mu \mathrm{g} / \mathrm{kg}$ [9]. This phenomenon is thought to be due to spinal anesthesia reaching maximum sensory block levels, with secondary hypotension augmented by the addition of the hypotensive effects of dexmedetomidine. The hypotensive effect of dexmedetomidine exhibited a dose-dependent pattern. On the other hand, the incidence of significant bradycardia was not dependent on the dose of dexmedetomidine, although it was relatively high in this study in comparison to the previous report in which the administered dose range of dexmedetomidine was consistent with that employed here [14]. Therefore, we assumed that the development of bradycardia in this study should be ascribed to the spinal anesthesia or loading injection of dexmedetomidine, as these are common factors to all three groups. Although the effects of loading dose on the dexmedetomidine-induced bradycardia remain to be elucidated, our assumption is supported by a meta-analysis, in which the risk of bradycardia associated with dexmedetomidine infusion was increased only in cases with a loading dose [15].

In the literature, the intended level of sedation using dexmedetomidine has been reported to be achieved at doses of 0.2$0.7 \mu \mathrm{g} / \mathrm{kg} / \mathrm{hr}[10]$. Within the recommended dose range, the current study suggests that the lowest dose may be appropriate for sedation during surgical procedures under spinal anesthesia. Such a conclusion drawn from this study may be associated with several factors, such as spinal anesthesia, age, and the defined RSS score as adequate sedation, which possibly influence the level of sedation or affect the titration of the dexmedetomidine dose. Spinal anesthesia has been reported to induce sedation and reduce anesthetic requirements, and a positive correlation has been exhibited between the depth of sedation and the extent of the block [16]. This effect is explained by the hypothesis of decrease in afferent sensory input with onsecutive reticulo-thalamo-cortical inhibition. Age is one of the factors influencing the level of sedation. The dose requirements for sedative agents are reportedly decreased in elderly patients [17]. Therefore, spinal anesthesia and the inclulsion of elderly patients in the current study might be contributing factors to the decreased requirement for dexmedetomidine. Finally, we have chosen the RSS score of 3 as it meets the condition of conscious sedation, which is a minimally decreased level of consciousness preserving the patients' ability to maintain their airway independently and to respond appropriately to physical stimulation or verbal command [18]. The incidence of inadequate sedation with RSS score below 3, thus necessitating midazolam administration, was comparable between the three groups and showed no statistically significant correlation with the dose of dexmedetomidine. Although statistically not significant, the RSS scores of group C after dexmedetomidine administration tended to be higher than those of group A or B. When taking into consideration that conscious sedation provides additional advantages including preserved airway reflex and rapid recovery, increased dose of dexmedetomidine may not offer any beneficial effects compared to low dose, such as the $0.25 \mu \mathrm{g} / \mathrm{kg} / \mathrm{hr}$ of the current study, in terms of adequate sedation. Collectively, the results of the current study clearly indicate that dexmedetomidine $1 \mu \mathrm{g} / \mathrm{kg}$ followed by continuous administration at an infusion rate of $0.25 \mu \mathrm{g} / \mathrm{kg} / \mathrm{hr}$ may be appropriate for sedation of patients receiving spinal anesthesia.

Although $\mathrm{SpO}_{2}$ values significantly decreased after dexmedetomidine administration compared to baseline values, clinically significant desaturation did not develop in the current study. This finding might be attributed to the following observations. First, dexmedetomidine has been reported to have a respiratorypreserving property $[19,20]$. In the study of Belleville et al. [20], which investigated the ventilatory effects of dexmedetomidine infusion in human volunteers, infusion of dexmedetomidine $2.0 \mu \mathrm{g} / \mathrm{kg}$ produced no episodes of significant arterial oxygen desaturation to below $90 \%$. According to their study, there was also no significant reduction in respiratory rate, albeit with mildly decreased tidal volume. The effects of dexmedetomidine on human respiration are known to be much less marked than those of opioids and other intravenous sedative agents, and they appear to be similar in order of magnitude to those seen in the heavy sleep state [5]. Second, dexmedetomidine is known to have an analgesic-sparing effect $[7,8]$. The inhibition of the locus ceruleus by dexmedetomidine results in disinhibition of the noradrenergic nuclei, thus norepinephrine, the most familiar and intensively-investigated transmitter implicated in descending inhibition of pain [21], might reduce spinal nociception [22]. In addition dexmedetomidine may bind to spinal $\alpha-2$ adrenoceptors to produce a potent analgesia [23]. The analgesic-sparing effect of dexmedetomidine could potentially reduce postoperative opioid consumption $[7,8]$, which might in turn decrease the risk of developing respiratory depression in the PACU in the current study. Third, the lack of respiratory depression observed in the current study may be, at least in part, related to the study design that necessitated frequently assessing patients, thus stimulating the level of consciousness.

There are some limitations to this study. First, dexmedetomidine dose-dependently increased the development of hypotension but not that of bradycardia in the current investigation. Thus the differential effects of dexmedetomidine on the blood pressure and heart rate should be further investigated in a future study, particularly under spinal anesthesia. Second, because the doses of continuous intravenous dexmedetomidine in the current study were based on a previous study, we did not investigate the effect of doses lower than $0.25 \mu \mathrm{g} / \mathrm{kg} / \mathrm{hr}$. Third, we did not assess the differences among the groups regarding analgesic consumption in the PACU and the duration of sensory or motor blockade produced by spinal anesthesia. In a previous 
study, intravenous dexmedetomidine prolonged the time required for recovery from sensory and motor blockade [24]. Delayed recovery from spinal anesthesia may be beneficial in cases of surgery of long duration but harmful in the ambulatory care setting. Finally, we did not consider the effect of age in relation to sedative dose. Therefore, further studies are needed to determine the optimal sedative dose of dexmedetomidine that will provide both hemodynamic stability and adequate duration of spinal anesthesia.

In conclusion, supplementation of spinal anesthesia with intravenous dexmedetomidine produced an adequate level of sedation in all patients that enabled patient's cooperation without inducing significant respiratory depression. However, the development of hypotension was observed as a dose-dependent side effect of dexmedetomidine. We suggest that intravenous injection of dexmedetomidine $1 \mu \mathrm{g} / \mathrm{kg}$ followed by continuous administration at an infusion rate of $0.25 \mu \mathrm{g} / \mathrm{kg} / \mathrm{hr}$ may be an appropriate dose for providing adequate sedation while minimizing the risk of hemodynamic instability.

\section{References}

1. Brown DL. Spinal, epidural and caudal anesthesia. In: Miller's Anesthesia. 7th ed. Edited by Miller RD: Philadelphia, Churchill-Livingstone. 2000, pp 1492-8.

2. De Andres J, Valia JC, Gil A, Bolinches R. Predictors of patient satisfaction with regional anesthesia. Reg Anesth 1995; 20: 498-505.

3. Ebert TJ, Hall JE, Barney JA, Uhrich TD, Colinco MD. The effects of increasing plasma concentrations of dexmedetomidine in humans. Anesthesiology 2000; 93: 382-94.

4. Hall JE, Uhrich TD, Barney JA, Arain SR, Ebert TJ. Sedative, amnestic, and analgesic properties of small-dose dexmedetomidine infusions. Anesth Analg 2000; 90: 699-705.

5. Venn RM, Hell J, Grounds RM. Respiratory effects of dexmedetomidine in the surgical patient requiring intensive care. Crit Care 2000; 4: 302-8.

6. Herr DL, Sum-Ping ST, England M. ICU sedation after coronary artery bypass graft surgery: dexmedetomidine-based versus propofolbased sedation regimens. J Cardiothorac Vasc Anesth 2003; 17: 576-84.

7. Arain SR, Ebert TJ. The efficacy, side effects, and recovery characteristics of dexmedetomidine versus propofol when used for intraoperative sedation. Anesth Analg 2002; 95: 461-6.

8. Taghinia AH, Shapiro FE, Slavin SA. Dexmedetomidine in aesthetic facial surgery: improving anesthetic safety and efficacy. Plast Reconstr Surg 2008; 121: 269-76.

9. Bloor BC, Ward DS, Belleville JP, Maze M. Effects of intravenous dexmedetomidine in humans. II. Hemodynamic changes. Anesthesiology 1992; 77: 1134-42.

10. Mantz J. Dexmedetomidine. Drugs Today (Barc) 1999; 35: 151-7.

11. Ramsay MA, Savege TM, Simpson BR, Goodwin R. Controlled sedation with alphaxalone-alphadolone. Br Med J 1974; 2: 656-9.

12. Jorm CM, Stamford JA. Actions of the hypnotic anaesthetic, dexmedetomidine, on noradrenaline release and cell firing in rat locus coeruleus slices. Br J Anaesth 1993; 71: 447-9.

13. Candiotti KA, Bergese SD, Bokesch PM, Feldman MA, Wisemandle W, Bekker AY. Monitored anesthesia care with dexmedetomidine: a prospective, randomized, double-blind, multicenter trial. Anesth Analg 2010; 110: 47-56.

14. Venn RM, Bradshaw CJ, Spencer R, Brealey D, Caudwell E, Naughton C, et al. Preliminary UK experience of dexmedetomidine, a novel agent for postoperative sedation in the intensive care unit. Anaesthesia 1999; 54: 1136-42.

15. Tan JA, Ho KM. Use of dexmedetomidine as a sedative and analgesic agent in critically ill adult patients: a meta-analysis. Intensive Care Med 2010; 36: 926-39.

16. Gentili M, Huu PC, Enel D, Hollande J, Bonnet F. Sedation depends on the level of sensory block induced by spinal anaesthesia. Br J Anaesth 1998; 81: 970-1.

17. Schnider TW, Minto CF, Shafer SL, Gambus PL, Andresen C, Goodale DB, et al. The influence of age on propofol pharmacodynamics. Anesthesiology 1999; 90: 1502-16.

18. American Society of Anesthesiologists Task Force on Sedation and Analgesia by Non-Anesthesiologists. Practice guidelines for sedation and analgesia by non-anesthesiologists. Anesthesiology 2002; 96: 1004-17.

19. Jaakola ML. Dexmedetomidine premedication before intravenous regional anesthesia in minor outpatient hand surgery. J Clin Anesth 1994; 6: 204-11.

20. Belleville JP, Ward DS, Bloor BC, Maze M. Effects of intravenous dexmedetomidine in humans. I. Sedation, ventilation, and metabolic rate. Anesthesiology 1992; 77: 1125-33.

21. Millan MJ. Descending control of pain. Prog Neurobiol 2002; 66: 355-474.

22. Guo TZ, Jiang JY, Buttermann AE, Maze M. Dexmedetomidine injection into the locus ceruleus produces antinociception. Anesthesiology 1996; 84: 873-81.

23. Kanazi GE, Aouad MT, Jabbour-Khoury SI, Al Jazzar MD, Alameddine MM, Al-Yaman R, et al. Effect of low-dose dexmedetomidine or clonidine on the characteristics of bupivacaine spinal block. Acta Anaesthesiol Scand 2006; 50: 222-7.

24. Elcicek K, Tekin M, Kati I. The effects of intravenous dexmedetomidine on spinal hyperbaric ropivacaine anesthesia. J Anesth 2010; $24: 544-8$. 